\title{
Исследование влияния особенностей конструкции установки магнетронного распыления на электрические и оптические свойства пленок оксида индия-олова
}

\author{
(ㄱ Д.А. Кудряшов ${ }^{1}$, А.А. Максимова ${ }^{1,2}$, Е.А. Вячеславова ${ }^{1}$, А.В. Уваров ${ }^{1}$, И.А. Морозов ${ }^{1}$, \\ А.И. Баранов ${ }^{1}$, А.О. Монастыренко ${ }^{1}$, А.С. Гудовских ${ }^{1,2}$ \\ ${ }^{1}$ Санкт-Петербургский национальный исследовательский Академический университет им. Ж.И. Алфёрова \\ Российской академии наук, \\ 194021 Санкт-Петербург, Россия \\ ${ }^{2}$ Санкт-Петербургский государственный электротехнический университет „ЛЭТИ“ им. В.И. Ульянова (Ленина), \\ 197022 Санкт-Петербург, Россия \\ E-mail: kudryashovda@spbau.ru
}

Поступила в Редакцию 25 ноября 2020 г.

В окончательной редакции 7 декабря 2020 г.

Принята к публикации 7 декабря 2020 г.

\begin{abstract}
Показано влияние относительного расположения магнетрона и подложки на электрические и оптические свойства формирующегося слоя оксида индия-олова (ITO). Рассмотрены причины данного поведения и показана роль кислорода в возникновении неоднородности свойств пленок ITO. Показано, что в режиме роста без дополнительного добавления кислорода удельное сопротивление пленок ITO различается на порядок $\left((2-14) \cdot 10^{-2}\right.$ Ом $\cdot$ см) при различном расположении подложки на подложкодержателе вдоль радиуса в диапазоне 0-14 см. На спектрах поглощения при этом наблюдаются различия в форме коротковолновой области спектра. Добавление незначительного $\left(0.1\right.$ ст. см${ }^{3} /$ мин $)$ количества кислорода в рабочую камеру в процессе роста оксида приводит к значительному повышению однородности электрических и оптических свойств ITO.
\end{abstract}

Ключевые слова: магнетронное распыление, оксид индия-олова, тонкие пленки.

DOI: $10.21883 /$ FTP.2021.04.50741.9561

\section{1. Введение}

Оксид индия-олова (Indium Tin Oxide или ITO) широко применяется при изготовлении современных полупроводниковых приборов благодаря своим уникальным оптическим и электрическим свойствам. ITO является вырожденным полупроводником $n$-типа с шириной запрещенной зоны $\sim 4$ эВ [1]. В то же время он характеризуется низкими значениями удельного сопротивления $\left(\sim 10^{-4} \mathrm{OM} \cdot \mathrm{cm}\right)$ и при этом имеет слабое поглощение в видимом спектре оптического излучения. Данные обстоятельства способствовали его широкому применению в производстве таких приборов, как солнечные элементы, индикаторные экраны (TFT), оптические датчики, прозрачная электроника. Оксид индия-олова также можно найти в составе различных многослойных оптических покрытий [2].

Существуют различные способы формирования наноразмерных слоев ITO, однако наибольшее распространение получил метод магнетронного распыления [3] благодаря его невысокой стоимости, масштабируемости и воспроизводимости. К настоящему времени в литературе имеется большое количество исследований, посвященных разработке технологии формирования и исследованию свойств оксида индия-олова [4-7]. Известно, что электрические и оптические свойства тонких пленок ITO крайне чувствительны к стехиометрическому составу и структурным свойствам материала. Кристаллическая решетка ITO представлена тремя элементами: индий, олово и кислород, и только крайне узкий диапазон их соотношения (90\% $\quad \mathrm{In}_{2} \mathrm{O}_{3}$ и $\left.10 \% \quad \mathrm{SnO}_{2}\right)$ приводит к формированию материала с высокой проводимостью и слабым поглощением в видимой части оптического спектра [5,6]. Известно также, что в процессе магнетронного распыления мишени ITO указанного выше состава часть кислорода из материала мишени не достигает поверхности подложки, из-за чего необходимо дополнительно подавать кислород в рабочую камеру, причем в различных публикациях оптимальное значение парциального давления кислорода отличается [7-10]. Кроме того, имеются работы, где отмечается, что последующий отжиг оксида при температуре $\sim 200^{\circ} \mathrm{C}$ приводит к заметному улучшению его свойств $[11,12]$, хотя в других работах $[7,10,13]$ удавалось вырастить материал со схожими характеристиками и без дополнительного отжига. Причина некоторого разброса параметров рабочих процессов, приводящих к формированию ITO с оптимальными характеристиками, может быть связана с использованием установок магнетронного осаждения, различающихся конструкцией ростовой камеры. Типичная камера установки магнетронного распыления включает вращающийся металлический диск (подложкодержатель) и находящиеся на некотором расстоянии один или несколько магнетронов с 
закрепленными на них материалами для распыления (мишени). При этом расстояние от мишени до подложкодержателя и их взаимное расположение может значительно различаться в зависимости от конструкции установки. Облако плазмы рабочего газа над мишенью удерживается благодаря магнитному полю, напряженность которого также может варьироваться в зависимости от конструкции магнетрона. Высокоэнергетичные частицы газа, находящиеся в плазме, выбивают материал мишени в окружающее пространство камеры и в зависимости от формы плазменного облака траектория движения атомов индия, олова и кислорода может в значительной степени различаться, что может сказываться на стехиометрии формирующегося материала, а это приводит к изменениям его электронных и оптических свойств.

Таким образом, цель данного исследования заключается в изучении влияния расположения конструкционных частей камеры установки магнетронного распыления на свойства формирующегося оксида индия-олова.

\section{2. Детали эксперимента}

Для формирования пленок ITO использовалась установка высокочастотного магнетронного распыления Вос Edwards Auto 500 RF. В качестве мишени выступал $3^{\prime \prime}$ диск из спеченного оксида состава 90\% $\quad \operatorname{In}_{2} \mathrm{O}_{3}$ и $10 \% \quad \mathrm{SnO}_{2}$ толщиной $5 \mathrm{MM}$, изготовленный компанией LTS Chemicals (CША). Перед осаждением камера установки откачивалась до остаточного давления $5 \cdot 10^{-6}$ мбар. Аргон чистотой $99.999 \%$ и кислород 99.999\% поступали в камеру во время роста оксида со скоростью 12 и $0-0.2$ ст. см $3 /$ мин для аргона и кислорода соответственно. При этом рабочее давление в камере составляло $1.5 \cdot 10^{-3}$ мбар. Каждый образец формировался при фиксированной мощности на магнетроне в диапазоне 40-70 Вт.

В качестве подложки использовались предметные стекла, расположенные по прямой вдоль радиуса подложкодержателя. Таким образом, имитировалось различное расположение образцов относительно магнетрона. За один процесс удавалось вырастить серию образцов, расположенных на расстоянии 0-13 см от края подложкодержателя. При этом в данной установке центр мишени располагался на расстоянии 4 см от края подложкодержателя. Кратчайшее расстояние между мишенью и подложкодержателем составляло 100 мм.

Перед напылением производилась очистка подложек путем их кипячения в изопропиловом спирте. После промывки обильным количеством деионизованной воды подложки высушивались под струей азота. В процессе роста пленок ITO дисковый подложкодержатель радиусом 13 см вращался со скоростью 60 об/мин.

Толщины выращенных пленок ITO измерялись на профилометре AMBiOS XP-1. Поверхностное сопротивление пленок определялось четырехзондовым методом с помощью источника-измерителя Keithley 2400. Исследование оптических свойств тонких пленок проводилось при помощи спектрометра AvaSpec.

\section{3. Экспериментальные результаты}

Была изготовлена серия образцов, отличающихся расположением на подложкодержателе относительно его края, а также мощностью их формирования. На первом этапе для исключения влияния кислорода на свойства формируемого оксида в реакционную камеру подавался только аргон.

Как видно из рис. 1, при магнетронном распылении скорость роста пленок ITO ведет себя крайне нелинейно как в зависимости от мощности, так и от положения образца на подложкодержателе. В центре подложкожкодержателя и на его краях скорость роста заметно ниже, чем на участке 4-8 cм. В интервале мощности от 40 до 60 Вт наблюдается резкое увеличение скорости роста, переходящее потом в более плавное. При мощности 70 Вт в области над магнетроном начинают наблюдаться разряды плазмы, поэтому дальнейшее увеличение мощности было нецелесообразно.

На рис. 2 приведены результаты измерения поверхностного сопротивления $\left(R_{\mathrm{sh}}\right)$ пленок оксида индия, выращенных при различной мощности магнетрона. Очевидно, что наблюдается нелинейная зависимость $R_{\mathrm{sh}}$ от положения образца на подложкодержателе. Так, наибольшее различие в $R_{\mathrm{sh}}$ проявляется при низких мощностях магнетрона. Если, например, оксид, формирующийся при мощности 40 Вт, имеет максимальную неоднородность по толщине в 1.5 раза (см. рис. 1), то его поверхностное сопротивление различается в 6.5 раз. Таким образом, одним влиянием толщины на $R_{\mathrm{sh}}$ нельзя объяснить данное поведение. Удельное сопротивление тонких пленок ITO, рассчитанное из значений $R_{\text {sh }}$ и толщины оксида, в зависимости от расположения образца

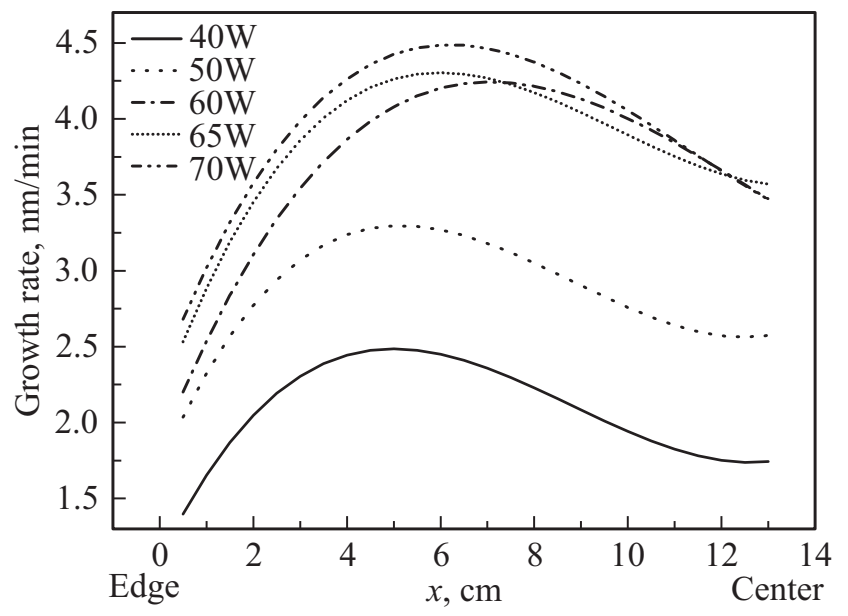

Рис. 1. Зависимость скорости роста пленок ITO от мощности магнетрона и положения образца на подложкодержателе. 


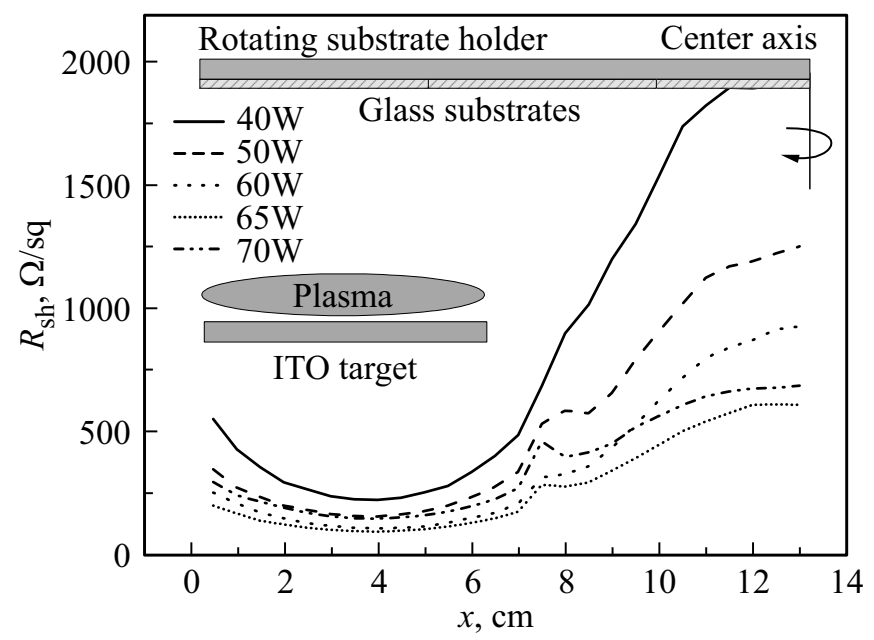

Рис. 2. Зависимость поверхностного сопротивления пленок ITO от мощности магнетрона и положения образца на подложкодержателе.

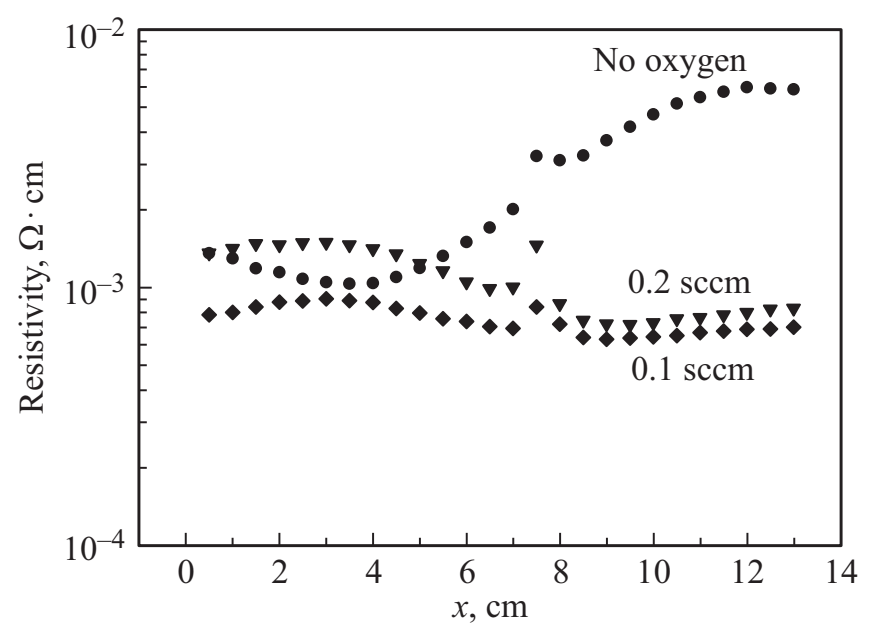

Рис. 3. Зависимость удельного сопротивления пленок ITO от потока кислорода в процессе роста и положения образца на подложкодержателе при мощности магнетрона 65 Вт.

на подложкодержателе показывает похожую динамику, что указывает на значительное различие в структурных свойствах пленок, расположенных на краю подложкодержателя и в его центре.

При введении в ростовую камеру незначительного количества кислорода $\left(0.1-0.2\right.$ ст. $\left.\mathrm{cm}^{3} / \mathrm{Mин}\right)$ при мощности магнетрона 65 Вт происходит слабое изменение скорости роста ITO. Вначале наблюдается подъем, затем скорость роста падает до значений, близких к уровню до введения кислорода. Тем не менее даже добавление этого количества кислорода приводит к резкому изменению удельного сопротивления пленок ITO (рис. 3). Одновременно со снижением величины удельного сопротивления происходит выравнивание данного параметра по отношению к расположению образца на подложкодержателе. Дальнейшее увеличение потока кислорода приводит к ожидаемому росту удельного сопротивления, причем в области на подложкодержателе, близкой к центру магнетрона, данный рост происходит сильнее.

На рис. 4 представлены графики спектральной зависимости коэффициента пропускания ITO от потока кислорода и положения образца на подложкодержателе для пленок, выращенных при мощности магнетрона 65 Вт. Толщина образцов, расположенных на расстоянии $4 \mathrm{~cm}$ от края подложкодержателя (рис. 4,a), составляла от 110 до 125 нм. При удалении от края на $13 \mathrm{~cm}$ (рис. $4, b)$ толщина пленок была меньше - от 95 до 115 нм. Из рис. 4 видно, что для случая отсутствия подачи кислорода в камеру во время роста пленки ITO коэффициент поглощения в коротковолновой области спектра различается для пленок, сформированных в разных частях подложкодержателя. Чем дальше образец располагается от магнетрона, тем большее поглощение в нем наблюдается, несмотря на меньшую толщину оксида.

Добавка кислорода на уровне $0.1 \mathrm{cт.}^{3} / \mathrm{Mин}$ приводит к значительному увеличению коэффициента пропускания в коротковолновой области. В этом случае
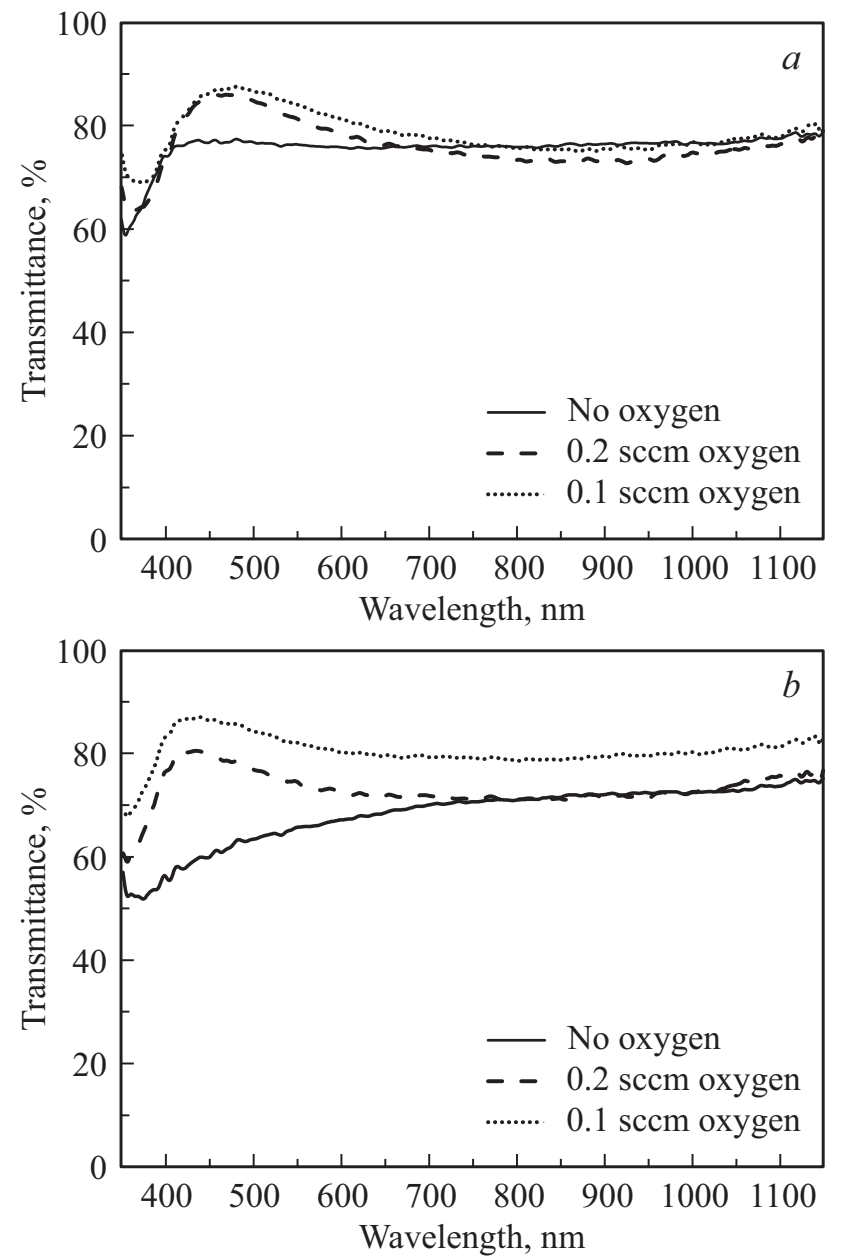

Рис. 4. Спектральная зависимость коэффициента пропускания от потока кислорода и положения образца на подложкодержателе на расстоянии $4(a)$ и $13 \mathrm{~cm}(b)$ от края подложкодержателя. 


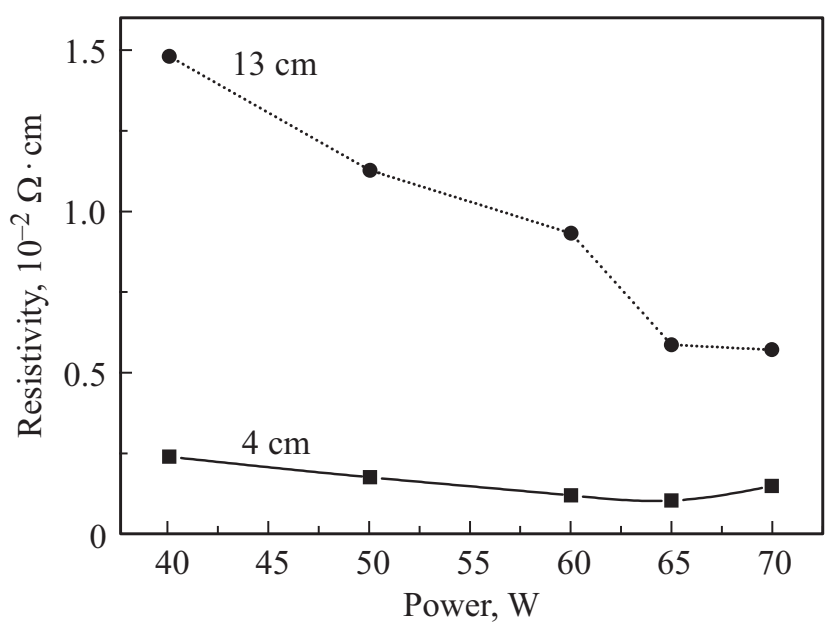

Рис. 5. Зависимость удельного сопротивления ITO от мощности магнетрона при фиксированном положении на диске (4 и 13 см от края).

спектральная зависимость коэффициента пропускания практически совпадает для образцов, расположенных на краю и в центре подложкодержателя. Такая же тенденция наблюдалась и для удельного сопротивления образца, выращенного в похожих условиях (см. рис. 5).

\section{4. Обсуждение результатов}

Приведенные зависимости измеренных характеристик ITO в зависимости от положения образца на подложкодержателе показывают, что оно играет существенную роль. Причем в случае бескислородного процесса различие по удельному сопротивлению может достигать одного порядка, а поглощение может различаться на несколько десятков процентов. Необходимо отметить, что в отличие от метода термического осаждения, в данном случае магнетрон нельзя рассматривать как точечный источник. В используемой установке над мишенью ITO в процессе ее распылении формируется тороидальное облако плазмы внешним диаметром $\sim 75$ мм. В зависимости от мощности, подаваемой на магнетрон, размеры данного облака меняются как в большую, так и в меньшую сторону. В плазме находятся высокоэнергетичные частицы, которые выбивают материал мишени в окружающее пространство камеры. В зависимости от формы плазменного облака траектория движения атомов индия, олова и кислорода может в значительной степени меняться, что видно из рис. 1, где, например, в центре подложкодержателя скорость роста осаждаемого материала практически не меняется с увеличением мощности в диапазоне 60-70Вт. В то же время в центре магнетрона на участке 4-6 см наблюдается значительный подъем скорости роста.

Нелинейный характер зависимости скорости осаждения от мощности магнетрона (см. рис. 1) может быть обусловлен нелинейной зависимостью напряжения смещения на обкладках магнетрона от подаваемой на магнетрон мощности. Однако, согласно измеренным данным, напряжение смещения в диапазоне 40-70 Вт ведет себя достаточно линейно. С другой стороны, при низких значениях мощности энергии частиц в газе может быть недостаточно для отрыва атомов материала с поверхности мишени, и тогда данная стадия будет лимитирующей. При достижении критического значения мощности ее дальнейшее повышение не будет давать столь существенный вклад в увеличение скорости роста пленки.

Кроме того, интересным является сильная нелинейная зависимость поверхностного сопротивления $R_{\mathrm{sh}}$ от положения образца на подложкодержателе и от мощности магнетрона. Наименьшее значение $R_{\mathrm{sh}}$ наблюдается при расположении образца прямо напротив центра магнетрона. При этом $R_{\mathrm{sh}}$ слабее зависит от значения мощности, чем при его отдалении от центра. Можно было бы предположить, что толщина оксида в области, близкой к центру магнетрона, больше, как показано на рис. 1, однако рассчитанные значения удельного сопротивления показывают похожую зависимость. Согласно данным работ [14-16], удельное сопротивление ITO определяется концентрацией структурных дефектов, в основном кислородными вакансиями. Кинетическая энергия вылетающих из материала мишени частиц различна в разных направлениях [3], поэтому на ортогональное направление приходится наибольший их поток и наибольшая энергия. По мере удаления от данного направления энергия частиц снижается.

Увеличение мощности магнетрона приводит к увеличению кинетической энергии, что до определенного момента обеспечивает их лучшее встраивание в кристаллическую решетку. Однако дальнейшее увеличение мощности будет способствовать слишком быстрому осаждению материала, во время которого атомы не будут успевать занять свои позиции в кристаллической решетке [12].

При одном и том же положении образца на подложкодержателе зависимость удельного сопротивления пленки ITO от мощности нелинейна (рис. 5). Для образца, расположенного напротив магнетрона, наблюдается минимум на графике при значении мощности $65 \mathrm{BT}$, в то время как с отдалением образца от края подложкодержателя резко повышается значение удельного сопротивления, а положение минимума смещается в область больших мощностей.

Таким образом, для бескислородного режима роста ITO с использованием установки Вoc Edwsards Auto $500 \mathrm{RF}$ наименьшее значение удельного сопротивления $\left(1 \cdot 10^{-3}\right.$ Ом $\cdot$ см $)$ будет у образца, расположенного напротив магнетрона, выращенного при мощности 65 Вт и давлении в камере $1.5 \cdot 10^{-3}$ мбар.

В пленках оксида индия-олова удельное сопротивление зависит от его структурных свойств. Согласно литературным данным, для пленок оксида индия-олова 
снижение концентрации носителей может объясняться двумя факторами: уменьшением количества кислородных вакансий, выступающих донорами, и увеличением числа двухвалентных атомов In и $\mathrm{Sn}$, являющихся акцепторами $[10,12]$.

Пленки ITO, осажденные при комнатной температуре на стекло или полимерные материалы, аморфны по структуре, и в таком случае их проводимость почти полностью определяется кислородными вакансиями (доноры) [17]. При этом наблюдается сильная зависимость проводимости от парциального давления кислорода. В отдельных случаях, при нехватке кислорода при осаждении материала, наблюдается так называемый черный субоксид на поверхности, когда трехвалентный индий в процессе распыления переходит в двухвалентный [12]. На рис. $4, b$ отчетливо видно сильное поглощение в коротковолновой области, которое уменьшается с добавлением кислорода.

Увеличение парциального давления кислорода приводит к тому, что $\mathrm{Sn}^{4+}$ захватывает дополнительный атом кислорода, формируя нейтральный кластер $\left(\mathrm{SnO}_{2}\right)_{2}$, где захваченный атом кислорода играет роль ловушки электронов. Из-за этого снижается подвижность носителей заряда и соответственно удельное сопротивление материала [10].

\section{5. Заключение}

Таким образом, представленные данные свидетельствуют о значительном влиянии расположения образца относительно магнетрона на характеристики формируемого материала. При анализе литературных данных при определении оптимальных режимов роста ITO необходимо также обращать внимание на относительное расположение образца относительно конструкции ростовой камеры. Добавление кислорода в камеру в ходе процесса роста пленок ITO приводит к значительному повышению однородности электрических и оптических свойств.

\section{Финансирование работы}

Представленные в работе исследования осуществлены в рамках проекта № 0791-2020-0004, поддержанного Министерством науки и высшего образования РФ.

\section{Конфликт интересов}

Авторы заявляют, что у них нет конфликта интересов.

\section{Список литературы}

[1] H. Kim, C.M. Gilmore, A. Piqué, J.S. Horwitz, H. Mattoussi, H. Murata, Z.H. Kafafi, D.B. Chrisey. J. Appl. Phys., 86 (11), 6451 (1999).

[2] V.S. Kaluba, K. Mohamad, P. Ferrer. Appl. Energy, 257, 114020 (2020).

[3] S. Swann. Phys Technol., 19 (2), 67 (1988).
[4] J.H. Shin, S.H. Shin J.I. Park, H.H. Kim. J. Appl. Phys., 89 (9), 5199 (2001).

[5] M.M. Aliyu, S. Hossain, J. Husna, N. Dhar, M.Q. Huda, K. Sopian, N. Amin. Proc. 38th IEEE Photovoltaic Specialists Conf. (Austin, TX, USA, 2012) p. 002009.

[6] W. Deng, T. Ohgi, H. Nejo, D. Fujita. Jpn. J. Appl. Phys., 40, 3364 (2001).

[7] D. Kudryashov, A. Gudovskikh, K. Zelentsov. J. Phys.: Conf. Ser., 461, 012021 (2013).

[8] S. Peng, X. Cao, J. Pan, X. Wang, X. Tan, A.E. Delahoy, K.K. Chin. J. Electron. Mater., 46 (2), 1405 (2017).

[9] Handbook of Thin Films, ed by H. Singh Nalwa. V. 5 (Toronto-London-N.Y., Academic Press, 2001) p. 3451.

[10] W.-F. Wu, B.-Sh. Chiou. Semicond. Sci. Technol., 11, 196 (1996).

[11] K. Samadzamini, J. Frounchi, H. Veladi. J. Korean Phys. Soc., 59 (5), 3280, (2011).

[12] S. Ishibashi, Y. Higuchi, Y. Ota, K. Nakamura. J. Vac. Sci. Tech. A, 8, 1403 (1990).

[13] F. Kurdesau, G.S. Khrypunov, A.F. da Cunha, M. Kaelin. J. Non-Cryst. Sol., 352, 1466 (2006).

[14] G.B. González, T.O. Mason, J.P. Quintana. J. Appl. Phys., 96, 3912 (2004)

[15] J.-H. Hwang, D.D. Edwards, D.R. Kammler, T.O. Mason. Solid State Ionics, $129(1-4), 135$ (2000).

[16] H.-N. Cui, V. Teixeira, L.-J. Meng, R. Martins, E. Fortunato. Vacuum, 82, 1507 (2008).

[17] Handbook of Visual Display Technology, ed. by J. Chen, W. Cranton, M. Fihn (Springer Verlag, Berlin-Heidelberg, 2012) p. 2700.

Редактор А.Н. Смирнов

\section{Study of the influence of design features of a magnetron sputtering chamber on the electrical and optical properties of indium-tin oxide films}

D.A. Kudriashov ${ }^{1}$, A.A. Maksimova ${ }^{1,2}$, E.A. Vyacheslavova' ${ }^{1}$, A.V. Uvarov' ${ }^{1}$, I.A. Morozov', A.I. Baranov ${ }^{1}$, A.O. Monastyrenko ${ }^{1}$, A.S. Gudovskikh ${ }^{1,2}$

${ }^{1}$ Alferov University, 194021 St. Petersburg, Russia

2 St. Petersburg Electrotechnical University „LETI", 197022 St. Petersburg, Russia

Abstract The influence of the relative position of the magnetron and the substrate on the electrical and optical properties of the forming indium tin oxide (ITO) layers is demonstrated. The reasons for this behavior are considered and the role of oxygen in the onset of inhomogeneity of the properties of ITO films is shown. It is shown that, in the growth mode without additional oxygen addition, the resistivity of ITO films differs by an order of magnitude $\left((2-14) \cdot 10^{-2} \mathrm{Ohm} \cdot \mathrm{cm}\right)$ for different positions of the substrate on the substrate holder along the radius in the range of $0-14 \mathrm{~cm}$. In this case, absorption spectra are observed differences in the shape of the short-wavelength region of the spectrum. The addition of an insignificant $(0.1 \mathrm{sccm})$ amount of oxygen to the working chamber during the growth of the oxide leads to a significant increase in the homogeneity of the electrical and optical properties of ITO. 